\title{
Wavelet filtering in high speed and high precision acquisition system
}

\author{
Liping Yuan
} School of Information Engineering Wuhan University of Technology Huaxia College, Wuhan,
430000, China

Keywords: acquisition systems, digital filtering, wavelet.

\begin{abstract}
High-speed high-precision data acquisition applications in modern industrial control field is very wide. The reasons of Environmental and circuit, the interference signal is introduced into the collection process inevitably, resulting in loss of precision and sampling error rate increases. When improving hardware near extreme conditions, using wavelet filtering software algorithm to eliminate interference and improve the accuracy of the sampling are widely carried out. Based on the 10-channel 16-bit sampling system, wavelet filtering effect is verified and quantified to prove the validity of wavelet filtering to improve the accuracy of the sampling.
\end{abstract}

\section{Introduction}

Wavelet transform as a means of engineering signal processing are causing concern and attention of researchers. Geophysical survey [1], the seismic survey [2], mechanical fault diagnosis [3] has been widely used in many fields. In recent years, wavelet applications in high-speed high-precision data acquisition and more widely. Accurate or not affect data collection is mainly due to the process of obtaining the data through the hardware circuit, as long as there exists a circuit where there are interference and noise, cause the data acquisition errors, thus affecting the degree of precision. Especially in the case of relatively high precision, even a small error, it will have a great impact on Subsequent data analysis, precision control field analysis never allow to happen. Digital filtering can be done Simulation filtering unmatched precision.

Article 16 for a high-precision acquisition system in Mat lab environment using wavelet techniques to collect raw data processing, and quantitative analysis of the results, in order to verify the effectiveness of the wavelet filtering algorithm to improve the accuracy of the sampling.

\section{Acquisition System}

This article is designed data acquisition system, the input voltage range of plus or minus $10 \mathrm{~V}$, the need to complete the 10-channel signal acquisition work, which requires a sampling rate of not less than 1.25MS / s, 16-bit resolution, system integration error $2 \mathrm{mv}$.

Voltage range of the original signal plus or minus $10 \mathrm{~V}$, due to the sampling chip cannot handle negative voltage, so that the front of the need for signal conditioning, after front-end processing circuit, the voltage changes to meet the range of A / D sampling chip processed voltage range. The reason to join the digital isolation, because the sampling output signal after A / D sampling chip after slightly, and used for the control of FPGA's noisy, if not digital isolation, FPGA noise will be on the sampling results caused a very big impact, thus affecting the accuracy. FPGA chip is used for sampling and Ethernet transmission control module. FPGA After receiving the sample data, the data will be cached, and then wait for a certain number of accumulated data, by controlling the Ethernet data encapsulated into Ethernet frame format, sent to the host computer, the PC and then based on the data in the package when added to the flag 10 distinguish data signals, and then the original data by digital filtering algorithm, using the processed data to draw the sample waveform.

\section{Wavelet filtering algorithm to achieve}

Real-time wavelet filtering process, have to pay special attention to several key factors, which is critical for real-time wavelet filtering algorithm design, appropriate Wavelets and reasonable 
threshold function, scientific and suitable threshold decomposition scale [4- 6].

\section{Wavelet choice}

Haar and Daubechies orthogonal wavelets due to its unique advantages and compactly supported, and thus it is widely used in real-time de-noising occasions. Under normal circumstances, you can visually and popular form described by the matrix of linear orthogonal transform and its inverse transform their process of finite discrete convolution huge contribution to its solution results, which is particularly suited to limited resources and the use of cable programming computing microcontroller applications. Some choice will be easier, but according to the actual experience of tens of hundreds of show production time, Daubechies wavelet second or third order vanishing moments because of the unique advantages in practical application has been successful in a wide range of de-noising.

\section{Wavelet maximum depth and width of the sliding window}

Wavelet algorithm in another important concept is the maximum width of a sliding window of data, its metric is the number of window covering data $\mathrm{n}$. Select $\mathrm{n}$ principles are the following.

(1) the target data to be analyzed in a single cycle, if the range of the lowest frequency components of $\mathrm{m}$ sampling data can be obtained , $\mathrm{n}$ may be selected as an integer power of 2 , the minimum is usually taken.

(2) analysis of the target sequence being able to describe the situation with a certain order to become moving average model, an adaptive finite impulse response can be thoroughly clean way, the way of selecting the first case the same security.

(3) In general real-time system with limited resources, the resulting data is updated every few cycles, this time n selection just like the previous case is not the same, it is required to use this approach to ensure that the maximum target data. As can be seen, if it is multi-scale transformation, the use of a real-time system data within the computing resources available to update cycle, to ensure the completion of the maximum amount of data wavelet noise reduction processing $\mathrm{n}$. Obviously, when using multi-resolution wavelet transform, $n$ should be an integer multiple of a power of 2.

\section{Wavelet decomposition and reconstruction}

Smaller spatial concepts from the perspective of multi-resolution characteristics do explain vividly and gives signal into different frequency channels algorithms and reconstruction algorithms.

(1) Decomposition algorithm

If $\mathrm{X}$ as a discrete signal to be decomposed, according to the decomposition algorithm,

$\left\{\begin{array}{l}a_{j+1}=H a \\ b_{j+1}=G b_{j}\end{array}\right.$

Which range from 0 to $\mathrm{j}$, $\mathrm{J}$ represents the highest decomposition level, $\mathrm{H}$ representative lowpass filter; $\mathrm{G}$ stands for high-pass filter, and represent the low and high frequency signals of the original signal in the next. Is the original signal in different frequency bands of adjacent components? Will eventually be decomposed signal decomposition and. The decomposition algorithm is the decomposition algorithm uses two extraction, so the length of each decomposed signal data than halved before decomposition, leaving the total length of the output data is consistent with the input data length. Reducing the number of signal prediction is detrimental, but after the signal Mallet decomposition algorithm can be reconstructed after the Second interpolation reconstruction algorithm.

reconstruction algorithm:

$a_{j}=H^{*} a_{j+1}+G^{*} b_{j+1}$

The expression $\mathrm{H}$ and $\mathrm{G}$ is a dual operator. With this type of decomposition of the signal can be reconstructed to increase the number of signals. And were reconstructed, $b_{1}, b_{2}, \ldots, b_{J}$ and $a_{J}$ get there.

$$
X=B_{1}+B_{2}+\ldots+B_{J}+C_{J}
$$

Which is a high-frequency signals of the first layer to the second layer of $\mathrm{J}$, $\mathrm{J}$ is the first layer of the reconstructed low frequency signals. In Mallet reconstruction algorithm, the low-frequency part 
typically contains the main information signal, the high frequency portion is associated with noise and disturbance. According to be analyzed, can continue to the low frequency part of the obtained re-decompose, thus also received signal is relatively high frequency signal and low-frequency part of the portion of the more

\section{Filtering effect Performance Evaluation}

\section{Low-frequency sampling filter effect}

Section When the sampling frequency of the system is relatively low, the filtering effect of four kinds of filtering algorithm is show below.

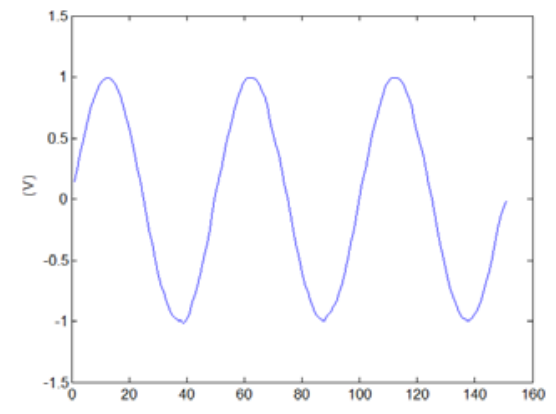

Fig. 1: weighted low-frequency filter

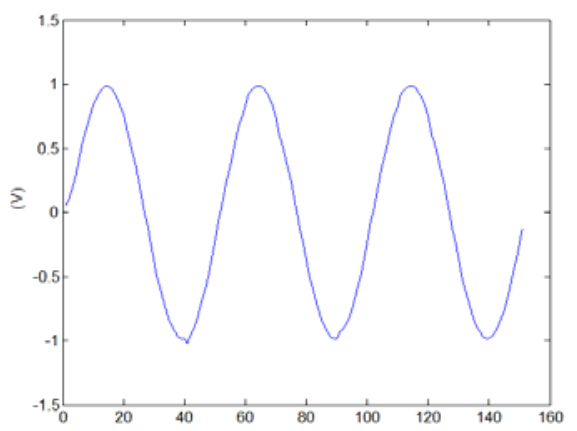

Fig. 2: a low-order lag filter

As can be seen, when the lower frequencies, the filtering effect of several algorithms have different levels of distortion exists, wavelet filtering algorithm and weighted recursive filtering approach works best, a first order lag filter the way, followed by the value filtering the worst way. This is because the median filtering approach is often used to process volatile demising some occasions, and we tested the use of an analog signal derived from the function signal generator, which signal is relatively stable, and therefore such a median filter signal filtering is no advantage, so more serious distortion. Overall, only the results of wavelet filtering algorithm and weighted recursive filtering is fairly smooth, you need to compare them further filtering effect in the case of high-frequency sampling.

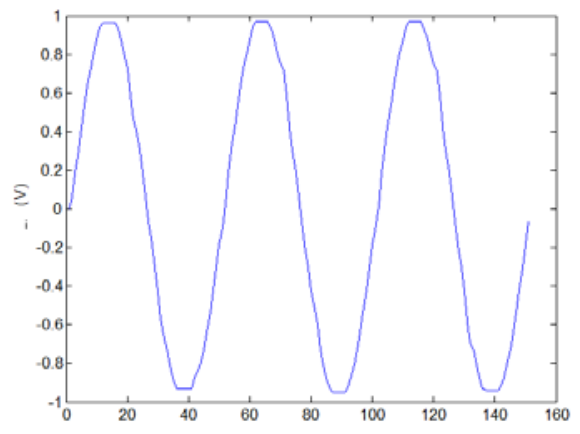

Fig. 3: the low-frequency filter by value 


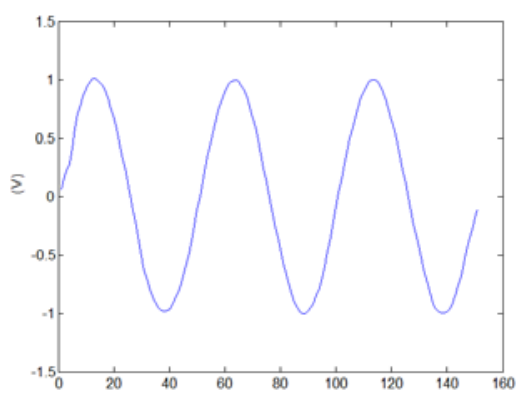

Fig. 4: a wavelet filter

\section{High-frequency sampling filter effect}

When increasing the sampling frequency of the sampling system, the filtering effect of four kinds of filtering modes are shown below.

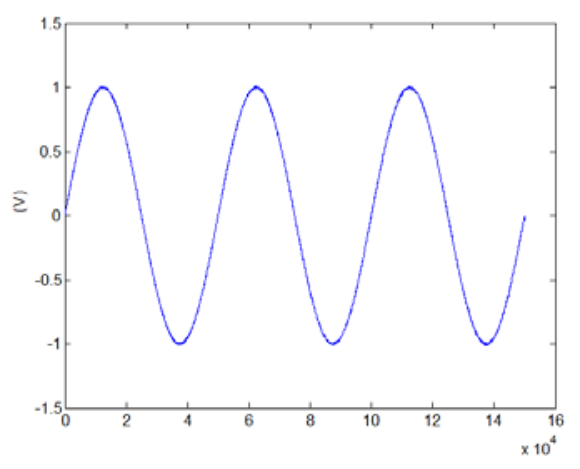

Fig. 5: Weighted frequency filtering

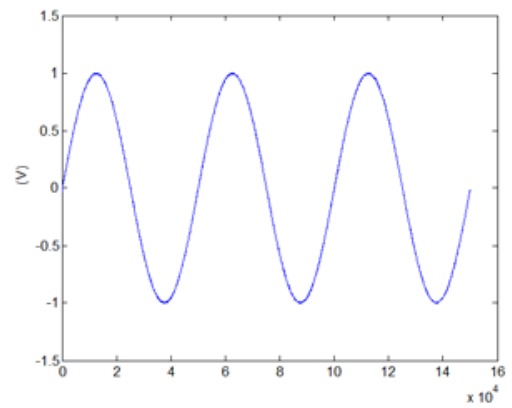

Fig. 6: an order lag frequency filtering

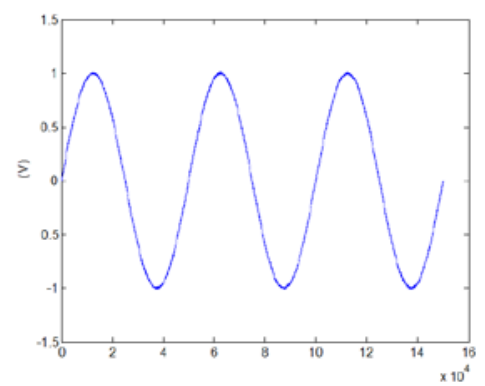

Fig. 7: Value frequency filtering 


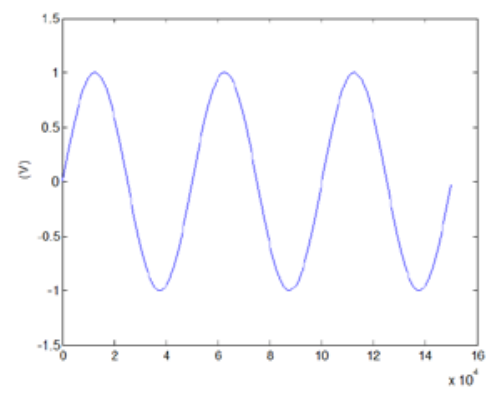

Fig. 8: wavelet high frequency filtering Methods

The simulation results can be seen from the above, the waveform smoothness and stability at high frequency wavelet filtering algorithm conditions are best. While the former three kinds of algorithms have a greater dispel interference, but the smoothness of the waveform does not achieve the desired results. If the first three filter system applied to the present system, when the system sampling rate, the processing effect of the algorithm is mainly the cause relatively large errors will occur when the host computer during the waveform drawing and perform other related processing.

\section{Quantitative Comparison}

This section uses the signal to noise ratio and the root mean square error to evaluate the filtering effect.

In order to compare the results of several more intuitive filtering approach, our original signal as a reference, the signal to noise ratio after the processed signal estimate (SNR) make the following definitions:

$$
S N R=10 \log \left[\frac{\sum_{k} x^{2}(k)}{\sum_{k}[x(k)-\hat{x}(k)]^{2}}\right]
$$

Mean square error (mse) is defined as

$$
M S E=\frac{1}{N} \sum_{n=1}^{N}[f(n)-\hat{f}(n)]^{2}
$$

Said the original signal, said after demising estimates of the signal. Which represents the original signal, after estimation signal indicates demising.

The higher the SNR based on knowledge, the original signal and the estimated signal, it is estimated that the signal with the original signal, the smaller the difference, the filtering effect is more ideal.

The following Table 4-1 and Table 4-2 respectively at two different frequencies of the respective statistical algorithms and the running time of the output signal to noise ratio, as follows:

Table 1: Sampling rate 1.5MHZ filtering performance when comparing different algorithms

\begin{tabular}{c|c|c}
\hline method & Output SNR (SNR) & error of mean square \\
\hline Wavelet filtering algorithm & 126.9262 & 0.0012 \\
\hline Average weighted recursive filtering & 97.3762 & 0.0054 \\
\hline The first-order lag filter & 87.2561 & 0.0090 \\
\hline median filter & 103.8942 & 0.0039 \\
\hline Table 2: Sample Rate 1.5KHZ filtering performance comparison of different algorithms \\
\hline method & Output SNR (SNR) & performance period(s) \\
\hline Wavelet filtering algorithm & 70.9102 & 0.0203 \\
\hline Average weighted recursive filtering & 40.6150 & 0.0925 \\
\hline The first-order lag filter & 41.8746 & 0.0868 \\
\hline median filter & 53.1845 & 0.6734 \\
\hline
\end{tabular}

By comparing the results can be seen, both in the case of high frequency or low frequency sampling, wavelet filtering algorithm output signal to noise ratio is the highest, the mean square error is minimal, indicating that the optimal wavelet filter has a filtering effect, so by comparing the system can justify the use of the wavelet filtering is feasible. 


\section{Conclusions}

In this paper, high-precision 10-channel 16-bit high-speed acquisition system, using four kinds of digital filtering algorithms include wavelet algorithm, including after the collected raw data sampled waveform processing reduction by Mat lab, and to quantify the results. The results show that the wavelet filtering algorithm to process the sample data, sampling can reduce noise and improve the accuracy of sampling, the sampling system can help improve the performance of the hardware in the same conditioned algorithm, the multi-objective bi-level programming problem is transformed into a single-level problem by using interpolation functions of the lower level solutions. The process avoids solving the lower level problems frequently, and reduces the computational cost. The major advantage of this algorithm is that it can solve some complex issues, in which the lower level problems are non-convex and non-differentiable. Hence, it can be used to deal with hard multi-objective bi-level programming problems.

\section{References}

[1] LuoYao, wang ping, Duan Shuling. Vertical aeromagnetic gradient to adjust T level method research [J]. Journal of geophysics, 2012 zhongguo kuangye daxue (11): 3854-3861.

[2] Li Hong-Nan, Sun Hong-Min. Application of Wavelet Analytical Method in Civil Engineering [J]. World Earthquake Engineering, 2003, 19(2): 16-22.

[3] Wang Jianbo, Luan Yuanzhong, Xu Junyi, et al. Wavelet analysis on data processing of bridge deformation monitoring [J]. Science of Surveying and Mapping, 2012, 37(3): 79-83.

[4] Chen wu every Yang Feng. Wavelet analysis and its application in image processing [M]. Beijing: science press, 2002.

[5] Ran Qi-Wen, Tan Li-Ying. Wavelet Analysis and Fractional Fouler Transform and its Application [M]. Defense Industrial Publish House, 2002. Farahi, M. H. \& Ansari, E. A new approach to solve muti-objective linear bilevel programming problems. Journal of Mathematics and Computer Science, 1(4), p.313-320,2010.

[6] Eman, O. E., Interactive-level multi-objective integer non-linear programming problem. Applied Mathematical Sciences, 5(65), 3221-3232, 2011. 